Check for updates

Cite this: RSC Adv., 2018, 8, 10673

Received 8th January 2018

Accepted 10th March 2018

DOI: $10.1039 / \mathrm{c} 8 \mathrm{ra00209f}$

rsc.li/rsc-advances

\title{
The Yxy colour space parameters as novel signalling tools for digital imaging sensors in the analytical laboratory $\dagger$
}

\begin{abstract}
Ashraf A. Mohamed, (D) * Ahmed A. Shalaby and Abdelnaby M. Salem
Digital imaging devices can be promising, sensitive, and cost-effective chemical sensors for resourcelimited settings. Three model colour reactions of iron were used and monitored using a simple platform consisting only of a camera, a cuvette, and a white paper diffuser. A desktop scanner and a mobile phone camera were also used as imaging devices. Captured images were analysed to obtain the RGB intensities (red, green, blue) and were further converted into the corresponding signals of the grayscale, CMY (cyan, magenta, yellow), XYZ (Y: luminance, XZ: chromaticity plane values) and Yxy colour spaces (Y: luminance, $x y$ : chrominance values) analytical signals. The elegant procedure utilizing the Yxy signals surpassed those based on RGB, grayscale, CMY and tristimulus XYZ data regarding the calibration graph linearity and detection limit and compare well with those data obtained from a sophisticated spectrophotometer for assessing iron in complex environmental samples. The simplicity, sensitivity and cost effectiveness of the approach make it suitable for poorly equipped laboratories and locally deprived communities.
\end{abstract}

\section{Introduction}

Spectrochemical methods utilizing the light absorbed by (or emitted from) a sample are very popular in quantitative chemical analysis. However, bulky size and relatively high cost are common disadvantages of many research grade spectrometers.

Therefore, methods of digital image-based analysis (DIBA) are suggested as simple and low-cost alternatives. DIBA relies on a colour change, wherein the analyte reacts with a chromogenic reagent to give a coloured product, the digital images of which are analysed to obtain the red, green and blue (RGB) intensity values $\left(I_{\mathrm{R}}, I_{\mathrm{G}}, I_{\mathrm{B}}\right.$, and $\left.I_{\mathrm{RGB}}\right)$. The RGB intensities, ${ }^{\mathbf{1 - 1 0}}$ the RGB absorbance $\left(A_{\mathrm{R}}, A_{\mathrm{G}}, A_{\mathrm{B}}, A_{\mathrm{RGB}}\right),{ }^{11-14}$ the normalized $I_{\mathrm{G}} / I_{\mathrm{R}}$ ratio, ${ }^{15}$ the cyan, magenta, and yellow (CMY) intensities, ${ }^{16-19}$ the tristimulus XYZ parameters, ${ }^{20-22}$ the greyscale intensity, ${ }^{23,24}$ and the inverted 8 bit greyscale intensity ${ }^{25,26}$ have been used as analytical signals in DIBA. Images have been captured using digital cameras, ${ }^{\mathbf{9}, \mathbf{1 4 , 1 8 , 2 4}}$ mobile phone cameras, ${ }^{\mathbf{4 , 7 , 1 2 , 1 5 , 1 7 , 1 9 , 2 3}}$ web cams,${ }^{10,11}$ or computer scanners. ${ }^{6,13,16,17,25,26}$ However, some other excellent papers on the topic have been undoubtedly missed and can be found in excellent reviews of the topic. ${ }^{27-29}$

The mathematical conversion of RGB intensities into the corresponding parameters of other colour spaces is well

Department of Chemistry, Faculty of Science, Ain Shams University, Abbassia, Cairo-11566, Egypt. E-mail: aamohamd@sci.asu.edu.eg; Fax: +202 24831836; Tel: +201001578849

† Electronic supplementary information (ESI) available: Additional figures and tables. See DOI: 10.1039/c8ra00209f documented; ${ }^{27-30}$ A brief introduction to the aforementioned colour spaces and their inter-conversions is given in the ESI. $\dagger$ However, some programs perform these conversions at a click of a button. The free online Colormine converter is very simple and was adopted in this work. ${ }^{31}$

It is worthy to mention that, the CIE (Commission Internationale de l'Eclairage, International Commission on Illumination) has classified the RGB, CMY, YXZ, and Yxy spaces as non-uniform colour spaces, where at constant luminance, the colour differences represented by equal distances are not perceived, by the human eye, as being equal. Moreover, the RGB and CMY are device dependent colour spaces that vary from one device to another and cannot represent all the colours. ${ }^{30}$

Despite the aforementioned progress, ${ }^{\mathbf{1 - 2 6}}$ the direct application of RGB intensity, ${ }^{1-10}$ RGB colour absorbance $,{ }^{11-14} \mathrm{CMY},{ }^{16-19}$ $\mathrm{XYZ},{ }^{20-22}$ and grayscale parameters, ${ }^{23-26}$ in analytical determinations exhibited a major disadvantage of poor linearity of its calibration graphs, especially at the low analyte concentrations that are commonly encountered in environmental samples. ${ }^{\mathbf{1 - 2 6}}$

Herein, we present the Yxy colour space parameters (Y: luminance, xy: chrominance values) as a novel signalling tool for digital imaging sensors in the analytical laboratory. The Yxy signals exhibited excellent performance compared to the RGB parameters and the corresponding data of a sophisticated Shimadzu UV 1601 PC spectrophotometer. Moreover, we show the advantages of Yxy over the RGB, grayscale, CMY, and tristimulus $\mathrm{XYZ}$ colour space signals regarding calibration graph linearity, slope-to-intercept ratio, and LOD. Also, we describe the design, use, and validation of a simple homemade 
platform and its application to the assessment of iron based on its reactions with 1,10-phenanthroline, 2,4,6-tris(2-pyridyl)-striazine (TPTZ) or salicylate. Our low-cost and simple platform is free from lenses, slits, mirrors, monochromators, photodiodes, photomultipliers, and signal amplifiers. It consists of (1) a digital camera, (2) a cuvette and (3) a white paper as a background diffuser. These advantages make the present platform an excellent successor to many of the closely related devices. ${ }^{1-26}$

\section{Experimental}

\subsection{Materials and reagents}

All reagents were of ACS grade and were used as received from Sigma-Aldrich (St. Louis, MO, USA) or Merck (Darmstadt, Germany). Ultrapure water and aqueous solutions were used throughout. 1,10-Phenanthroline, 2,4,6-tris(2-pyridyl)-s-triazine (TPTZ), sodium salicylate, ferrous ammonium sulphate hexahydrate, ferric ammonium sulphate dodecahydrate, and L-ascorbic acid were also used. Standard solutions for the determination of iron using phenanthroline, ${ }^{32-35} \mathrm{TPTZ},{ }^{35,36}$ and salicylate $^{37}$ were prepared according to standard/reference methods.

\subsection{Instruments and software}

Spectrophotometric measurements were performed on a Shimadzu UV 1601 PC Spectrophotometer (Kyoto, Japan) controlled by UVProbe-2.5 software. Eppendorf 10-100 and 100-1000 $\mu \mathrm{L}$ vary-pipettes (Westbury, NY, USA) and a calibrated EDT pH-mV meter (Dover Kent, UK) were also used.

Digital image-based analysis (DIBA) measurements were conducted using a simple homemade platform (spectrometer) consisting of (1) a cuvette as a sample container, (2) a digital camera as an imaging device, (3) white paper as a background diffuser. However, the sample container can also be a test tube, a beaker, or a microwell plate, and the digital imaging device can be a smartphone camera, a desktop scanner, or a simple webcam. The diffuser and the camera were fixed at $5 \mathrm{~cm}$ distances from both sides of the polylactic acid 3D-printed cellholder on a $20 \times 20 \mathrm{~cm}$ wooden plate, as shown in Fig. 1. For comparison of various digital imaging devices, images were captured using a 16.0 MP Canon PowerShot A810 digital camera, a 3.15 MP Sony Xperia E Dual smartphone camera, and the scanner of an HP LaserJet Pro M1536dnf MF printer.

A simple HP-Pavilion dv6-6176ex Notebook running Windows 8.1 was used for data treatment and analysis. Photoshop CC 2015.5 and ImageJ software version 1.51i were used for digital image cropping and RGB channel intensity calculations, respectively.

\subsection{Recommended procedure}

2.3.1. Procedure for iron determination using phenanthroline. An aliquot of the standard iron or sample solutions containing $\leq 80 \mu \mathrm{g}$ of $\mathrm{Fe}$ (III) or $\mathrm{Fe}(\mathrm{II})$ was transferred to a $25 \mathrm{~mL}$ volumetric flask. A $1 \mathrm{~mL}$ portion of each of the ascorbic acid, phenanthroline and acetic/acetate buffer solution ( $\mathrm{pH} 4.0)$ were added, and the flask was completed to the mark with water,

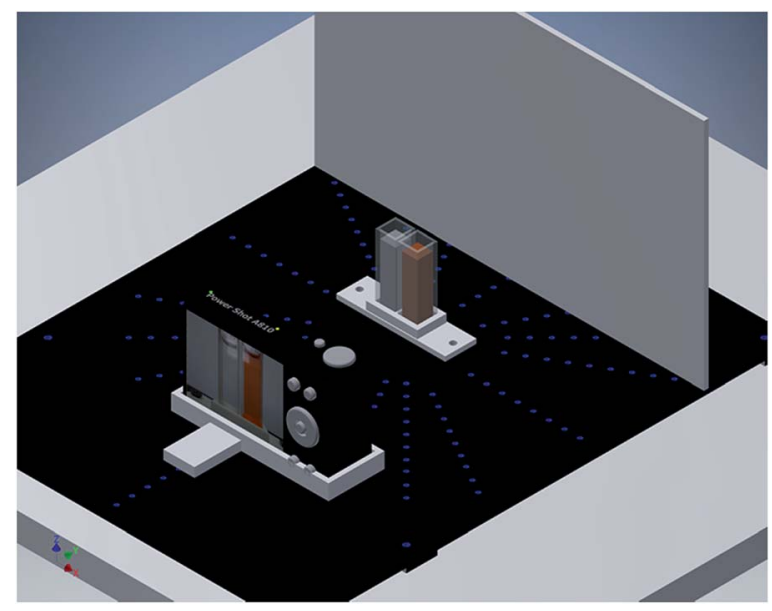

Fig. 1 The homemade platform design.

mixed well, and set aside for 15 min to allow for full colour development. ${ }^{32-35}$ A portion of the mixed solution was transferred to a $10 \mathrm{~mm}$ spectrophotometric cell, and the absorbance at $512 \mathrm{~nm}$ was measured against a similarly prepared blank. The same sample and blank cells were then transferred to our homemade platform to capture digital images using the Canon digital camera and the Sony smartphone. Another $250 \mu \mathrm{L}$ portion aliquot of the mixed solution was transferred to one chamber of a flat-bottom 96 microwell plate to take a scanned image.

2.3.2. Procedure for iron determination using TPTZ. An aliquot of the standard iron or sample solutions containing $\leq 50 \mu \mathrm{g}$ of $\mathrm{Fe}$ (III) or $\mathrm{Fe}(\mathrm{II})$ was transferred to a $25 \mathrm{~mL}$ volumetric flask. A $1 \mathrm{~mL}$ portion of each of ascorbic acid and acetic/ acetate buffer $(\mathrm{pH}=4.0)$ solutions were added followed by a $3 \mathrm{~mL}$ of TPTZ solution. The flask was completed to the mark with water, mixed well, and set aside for $5 \mathrm{~min}$ to allow full colour development. ${ }^{35,36}$ A portion of the reacting solution was transferred to a $10 \mathrm{~mm}$ spectrophotometric cell, and the absorbance at $595 \mathrm{~nm}$ was measured against a similarly prepared blank. Digital images were captured and processed as described above.

2.3.3. Procedure for iron determination using salicylate. An aliquot of the standard iron solution or sample solution containing $\leq 450 \mu \mathrm{g}$ of $\mathrm{Fe}(\mathrm{III})$ was transferred to a $25 \mathrm{~mL}$ volumetric flask. A $1 \mathrm{~mL}$ portion of each of $0.1 \mathrm{~mol} \mathrm{~L}^{-1}$ nitric acid and salicylate solutions was added, and the flask was diluted to the mark with water and then mixed will. A portion of the reaction solution was transferred to a $10 \mathrm{~mm}$ cell, and the absorbance at $525 \mathrm{~nm}$ was measured against a similarly prepared blank. ${ }^{37}$ Digital images were captured and processed as described above.

2.3.4. Pharmaceutical sample treatment for Fe-determination. A $1.00 \mathrm{~mL}$ sample or one tablet was transferred to a $50 \mathrm{~mL}$ beaker containing $30 \mathrm{~mL}$ of water and $5 \mathrm{~mL}$ concentrated $\mathrm{HCl}$. The mixture was boiled gently on a hotplate for $10 \mathrm{~min}$, cooled, filtered into a $100 \mathrm{~mL}$ volumetric flask, and diluted to the mark with water. A $10 \mathrm{~mL}$ aliquot of the resulting solution was diluted to $100 \mathrm{~mL}$ in a volumetric flask. ${ }^{32,35}$ 


\subsection{Procedure for digital image processing}

From each captured image, a representative homogenous area was cropped (a square of $354 \times 354$ pixels from the camera images, a square of $142 \times 142$ pixels from the smartphone images and a circle of 64,127 , and 253 pixels in diameter from the 300, 600 and 1200 dpi images of the scanner, respectively; however, any other predefined area can be used). Cropped images were processed to obtain the RGB channel intensities $\left(I_{\mathrm{R}}, I_{\mathrm{G}}\right.$, and $\left.I_{\mathrm{B}}\right)$, which can be used directly or after conversion into other colour spaces. For each image, the RGB colour absorbance $\left(A_{\mathrm{R}}, A_{\mathrm{G}}, A_{\mathrm{B}}\right.$, and $\left.A_{\mathrm{RGB}}\right)$ was calculated from $[A=$ $\left.\log \left(I_{0} / I\right)\right]$, where $I_{0}$ and $I$ are the channel intensities of the blank and sample, respectively. However, the Yxy colour absorbance was calculated from $\left[A_{x y}=\left|\log \left(I_{0} / I\right)\right|\right]$. The obtained RGB intensities were converted into the corresponding coordinates of other non-uniform colour spaces using the Colormine online converter. ${ }^{31}$

\section{Results and discussion}

\subsection{Preliminary studies}

The main advantages of the developed platform were its simplicity, ease of construction and high performance at minimum cost. Trials using conventional LED lamps as light sources ${ }^{4}$ were unsuccessful, as captured images were not homogeneous among their different parts, exhibiting variable degrees of brightness depending on not only the number and intensity of LED lamps but also the relative distances and angles between the lamp, cuvette, camera, and diffuser. Therefore, for the sake of simplicity, reproducibility, and cost reduction, we used the conventional fluorescent daylight lamp fixed to the ceiling of our laboratory (at about $2.5 \mathrm{~m}$ distance from the bench-top), as the light source. With this choice, the photo quality and the colour absorbance registered with the platform was almost independent of the lamp intensity, the relative platform-lamp position, camera-cuvette distance, and cuvette-diffuser distance, Fig. S1 and S2. $\dagger$ This is due to the relatively large distance between the lamp and cuvette that allowed a uniform light segment to reach the sample. However, for practical considerations, the camera and the diffuser were set at $5 \mathrm{~cm}$ distances from the 3-D printed cell holder carrying the cuvettes, Fig. 1. Moreover, the flash off mode of the camera was used to prevent any reflections on the cuvette surface due to the camera flash light.

\subsection{Comparison between RGB, grayscale, CMY, XYZ and Yxy parameters as signalling tools}

Our homemade platform was applied to the determination of iron using 1,10-phenanthroline, TPTZ and salicylate, which gave orange-red, blue-purple and violet colours, respectively. These reaction systems were selected to give three different colours covering a wide range of the visible spectrum. A representative area was cropped from each image (Fig. S2A-C) $\cdot \dagger$ These cropped images were analysed to obtain the corresponding RGB channel intensities. For the Fe(II)-phenanthroline system, plots of Fe(II) concentration $v s$. RGB intensities or their logarithmic values (i.e., $\log \left(I_{\mathrm{R}}\right), \log \left(I_{\mathrm{G}}\right), \log \left(I_{\mathrm{B}}\right)$, or $\left.\log \left(I_{\mathrm{RGB}}\right)\right)$, gave inadequate linearity, as shown in Fig. 2A and B. On the other hand, conversion of the RGB intensities to the corresponding 8 bit greyscale intensity and plotting $\mathrm{Fe}$ (II) concentration vs. greyscale intensity $\left(I_{\text {Grey }}\right)$, logarithmic greyscale intensity $\left(\log I_{\text {Grey }}\right)$ or greyscale colour absorbance ( $\left.A_{\text {greyscale }}\right)$ worsened the linearity, Fig. 2C-E.

To improve the linearity, we converted the RGB channel intensities into the corresponding RGB colour absorbance values $\left(A_{\mathrm{R}}, A_{\mathrm{G}}, A_{\mathrm{B}}\right.$, and $\left.A_{\mathrm{RGB}}\right)$, Fig. $2 \mathrm{~F}$, where inadequate linearity was also observed. Therefore, we tested the conversion of the RGB intensities into the corresponding colour absorbance values in the CMY, XYZ, and Yxy colour spaces, Fig. 2G-I. The conversion data clearly show that compared to the RGB, CMY and tristimulus XYZ values, the $x$ and $y$ coordinate of the Yxy space exhibited a marginal improvement not only in linearity $\left(R^{2}=0.999\right)$ but also in slope, slope-to-intercept ratio and detection limit (LOD $=0.07 \mathrm{mg} \mathrm{L}^{-1}$ ), Fig. 2I.

To confirm that these findings are not limited to the orangered coloured Fe-phenanthroline system, we examined the wellknown Fe(II)-TPTZ system exhibiting a deep blue-purple colour. Solutions containing $0.00-2.00 \mathrm{mg} \mathrm{L}^{-1} \mathrm{Fe}(\mathrm{II})$ were treated with TPTZ according to the recommended procedure. Images of these reacting solutions, Fig. S2B, $\dagger$ were captured, cropped, and analysed to obtain the RGB values and further converted into other non-uniform colour spaces' parameters. The relations between $\mathrm{Fe}(\mathrm{II})$ concentration and $I_{\mathrm{RGB}}, A_{\mathrm{RGB}}, A_{\mathrm{CMY}}, A_{\mathrm{XYZ}}$ and $A_{\mathrm{Yxy}}$, have been constructed. The data, Fig. S3A-E, $\dagger$ also showed that among all the tested signals, the $x$ and/or $y$ coordinate of the Yxy space exhibited marginal improvements in linearity $\left(R^{2} \geq 0.999\right)$, slope, slope-to-intercept ratio and detection limit $\left(\mathrm{LOD}=0.02 \mathrm{mg} \mathrm{L}^{-1}\right)$.

For further verification, we extended our work from the orange-red Fe-phenanthroline and the blue-purple Fe-TPTZ systems to the well-known violet-coloured $\mathrm{Fe}(\mathrm{III})$-salicylate system. Solutions containing $0.0-18.0 \mathrm{mg} \mathrm{L}^{-1} \mathrm{Fe}(\mathrm{III})$ were treated with salicylate according to the recommended procedure. Images of these reacting solutions, Fig. S2C, $\uparrow$ were similarly analysed and the relations between $\mathrm{Fe}(\mathrm{III})$ concentration and $I_{\mathrm{RGB}}, A_{\mathrm{RGB}}, A_{\mathrm{CMY}}, A_{\mathrm{XYZ}}$ and $A_{\mathrm{Yxy}}$, were established. Similarly, the data, Fig. S4A-E, $\dagger$ showed that among all the tested parameters, the $A_{\mathrm{y}}$ parameter of the Yxy space exhibited marginal improvements in linearity $\left(R^{2}=0.999\right)$, slope, slopeto-intercept ratio and detection limit.

In addition, the colour absorbance of the $x$ and/or $y$ coordinates of the Yxy colour space was compared to the absorbance readings recorded using the Shimadzu UV 1601 PC spectrophotometer for the three colour systems: Fe(II)-Phenanthroline, $\mathrm{Fe}(\mathrm{II})-\mathrm{TPTZ}$ and Fe(III)-salicylate, respectively, Fig. 3A-C. The regression parameters of Table $\mathrm{S} 1 \uparrow$ clearly show that colour absorbance data from digital images compete well with the corresponding spectrophotometric absorbance data, with the added advantage that our platform does not contain any monochromator, photomultiplier, signal amplifier, or any special optical unit.

Now, the challenging question is as follows: why do the $x$ and $y$ coordinate absorbances provide excellent linearity in the $\mathrm{Fe}(\mathrm{II})$ phenanthroline and Fe(II)-TPTZ systems, whereas only the $y$ coordinate absorbance exhibited excellent linearity in the Fe(III)- 

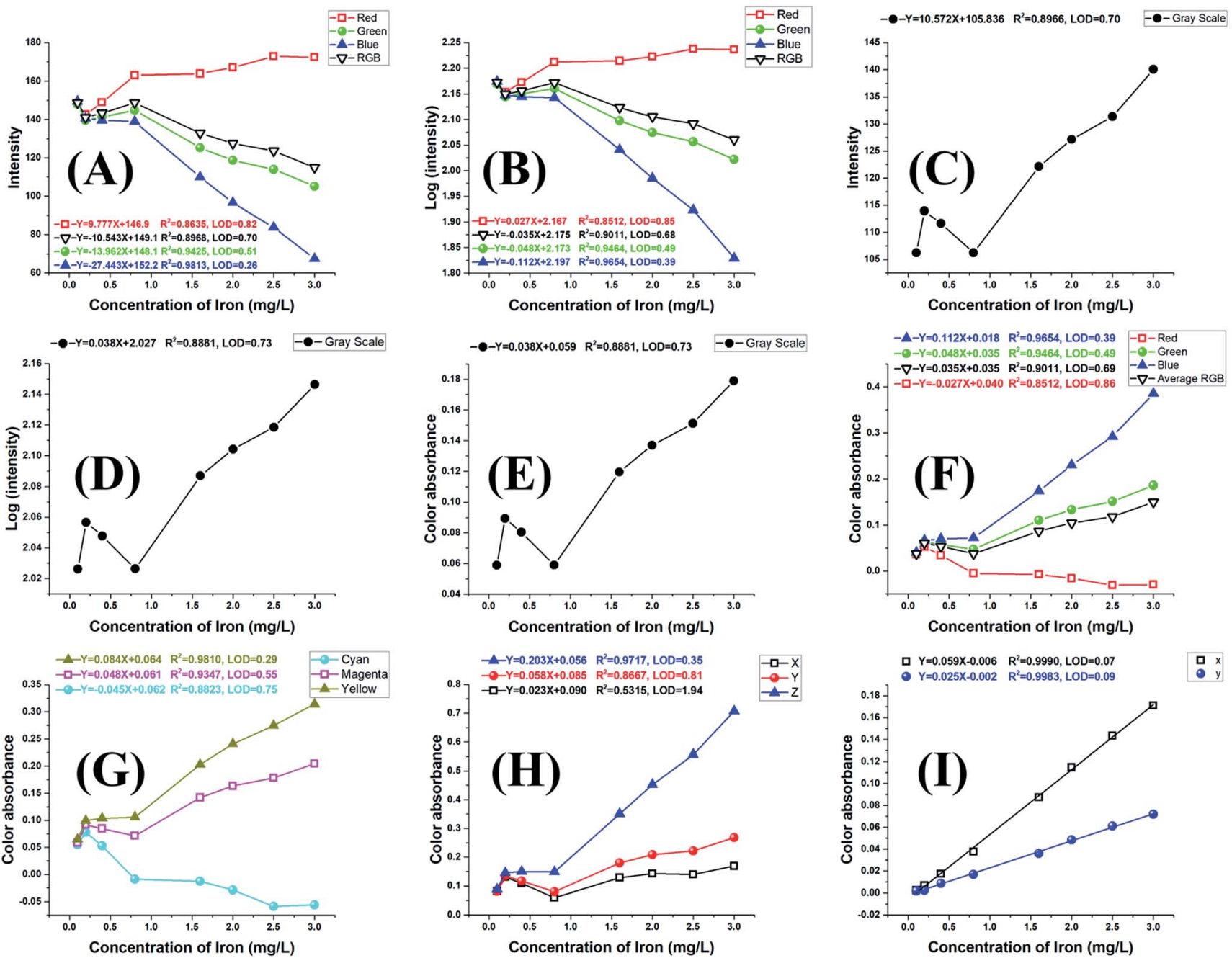

Fig. 2 Calibration graphs of Fe(॥)-phenanthroline solutions. Responses are RGB intensity (A), log /RGB (B), greyscale intensity (C), log(greyscale intensity) (D), greyscale colour absorbance (E), RGB colour absorbance (F), CMY colour absorbance (G), tristimulus XYZ colour absorbance (H), and $Y x y$ colour absorbance (I). Conditions are those of the recommended procedure.
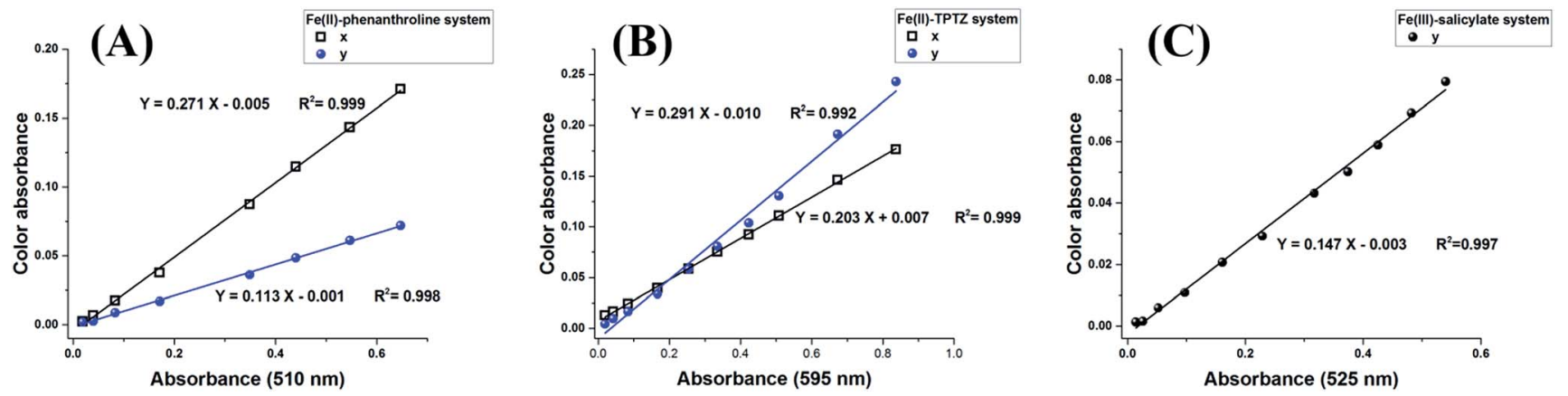

Fig. 3 Relationship between Yxy colour absorbance and spectrophotometric absorbance at the appropriate wavelength for the Fe(॥)-phenanthroline (A), Fe(II)-TPTZ (B), and Fe(III)-salicylate systems (C). The other conditions tested are those shown in Fig. 2.

Salicylate system? This can be explained by the fact that the $\mathrm{Fe}(\mathrm{II})$-phenanthroline and Fe(II)-TPTZ are orange-red and bluepurple, respectively, and are affected by both the $x$ and $y$ coordinate parameters. Therefore, they provide excellent calibration relations with both parameters. However, the $\mathrm{Fe}(\mathrm{III})$-salicylate system exhibits a violet colour and is mainly affected by the $y$ parameter; therefore, it gave excellent linearity with the $y$ parameter only.

It is worthy to mention that, according to the CIE, the XYZ and Yxy are device independent spaces and can represent all the 
colours because the xy gamut includes the RGB gamut inside it. However, the Yxy space has an advantage over the XYZ space as it normalizes the XYZ parameters and represents the colours in 2D plane that give good correlation with human vision attributes. $^{30}$ This may explain why the Yxy space data are more linear compared to other non-uniform colour spaces.

Based on the above findings, we can safely recommend using the $x$ and/or $y$ colour absorbance values of the Yxy space for analytical determination based on digital imaging. This is because the Yxy colour absorbance surpasses the conventional RGB intensities, greyscale intensity, log RGB, loggreyscale parameters, along with the colour absorbance values of RGB, greyscale, CMYK or tristimulus XYZ parameters.

\subsection{Comparison of different digital imaging devices}

To compare various image-capturing devices, we compared a Sony Xperia E smartphone with a 3.15 MP low-resolution

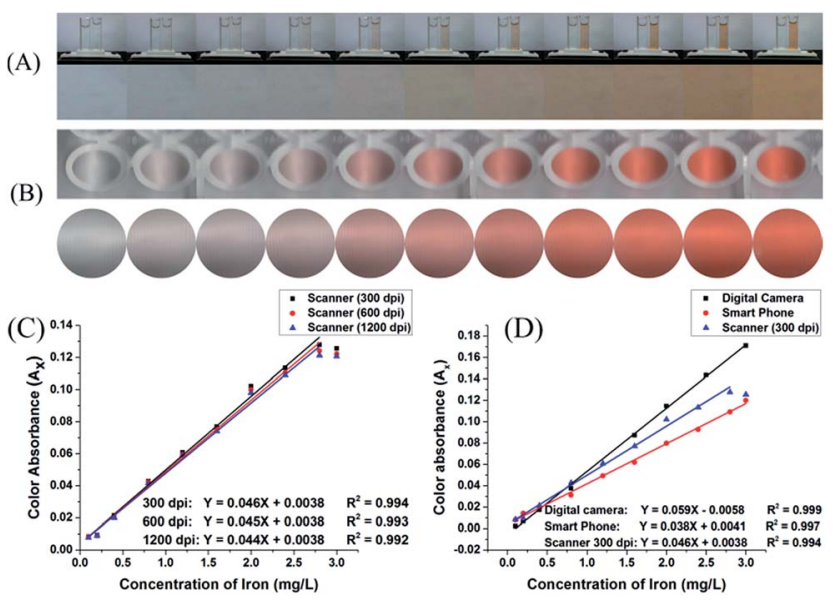

Fig. 4 Digital images and cropped images of Fe(॥)-phenanthroline standard solutions $\left(0.0-3.0 \mathrm{mg} \mathrm{L}^{-1} \mathrm{Fe}\right)$. Images were captured using (A) a Sony Xperia E smartphone equipped with a 3.15 MP camera and (B) the scanner of HP LaserJet Pro M1536dnf MF printer at $1200 \mathrm{dpi}$. Calibration graphs based on images from the desktop scanner at various pixel densities (C), and from various digital image-capturing devices (D). Captured images were arbitrarily compressed to fit into the page margins; however, for image processing, the original uncompressed images were used. The other conditions tested are those shown in Fig. 2. camera and the scanner of HP LaserJet Pro M1536dnf MF printer to our Canon PowerShot A810 digital camera with its 16 MP sensor. Digital images and the corresponding cropped areas of Fe(II)-phenanthroline solutions in $10 \mathrm{~mm}$ glass cells and 96microwell plates, were recorded, Fig. $4 \mathrm{~A}$ and $\mathrm{B}$. The relation between $\mathrm{Fe}(\mathrm{II})$ concentration and the $x$-coordinate colour absorbance $\left(A_{x}\right)$ of the scanner images at 300, 600 and $1200 \mathrm{dpi}$ were depicted, Fig. $4 \mathrm{C}$. The regression parameters clearly show that there is no significant difference in the linearity or slopes of the three calibration lines. Thus, to save time, we recommend using the $300 \mathrm{dpi}$ scan speed. The use of the microwell plate and scanner added the advantage of the possible direct analysis of samples in the form of thin-films, $\mathrm{nL}$ or $\mu \mathrm{L}$ droplets.

The relations between $\mathrm{Fe}(\mathrm{II})$ concentration and the colour absorbance $\left(A_{x}\right)$ obtained by the three image-capturing devices are depicted, Fig. 4D. The linear calibration equations indicate that the sensor sensitivity has the order digital camera > scanner $>$ smartphone, as evidenced from the slope values of $0.059>0.046>0.038$. However, the linearity is not significantly affected by changing the image-capturing device resolution, as all devices resulted in a correlation coefficient $R^{2} \geq 0.994$.

Similarly, the relation between the Fe concentration and $x$ or $y$ colour absorbance of images of Fe(II)-TPTZ and Fe(III)-salicylate solutions captured using various image-capturing devices, Fig. S5 and S6. $\dagger$ For both systems, the regression equations show no significant difference between the linearity and sensitivity of the different calibration lines constructed using the scanner at various levels of pixel density. Moreover, all calibration lines constructed using the three image-capturing devices show excellent linearity $\left(R^{2}>0.99\right)$, with the digital camera exhibiting the best sensitivity (slope).

\subsection{Determination of iron in real samples}

The performance of our homemade platform with the Yxy signalling was tested by its application to the assessment of iron in real samples. The analytical data obtained by the proposed approach were validated by comparison with the corresponding data obtained with the sophisticated Shimadzu UV 1601 PC spectrophotometer. For sample analysis, the digital camera was used as the image-capturing device, and the $A_{x}$ colour absorbance of the Yxy colour space was used as the analytical signalling tool. The 1,10-phenanthroline method was used for the

Table 1 Results of iron determination in real samples using the proposed DIBA and spectrophotometric methods

\begin{tabular}{|c|c|c|c|c|c|}
\hline & \multicolumn{2}{|c|}{ Iron concentration } & \multirow{2}{*}{$\begin{array}{l}F_{0.05}(3,3), \\
F_{\text {tabulated }}=39\end{array}$} & \multirow{2}{*}{$\begin{array}{l}\left|t_{0.05}(3,3)\right| \\
T_{\text {tabulated }}=2.78\end{array}$} & \multirow{2}{*}{$\begin{array}{l}P \text { value, } \\
P_{\text {tabulated }}=0.050\end{array}$} \\
\hline & DIBA & Spectrophotometry & & & \\
\hline Nile river water $\left(\mathrm{mg} \mathrm{L}^{-1}\right)^{a}$ & $0.52 \pm 0.02$ & $0.53 \pm 0.02$ & 1 & 0.61 & 0.573 \\
\hline Waste water $\left(\mathrm{mg} \mathrm{L}^{-1}\right)^{b}$ & $1.95 \pm 0.03$ & $1.94 \pm 0.02$ & 2.25 & 0.48 & 0.656 \\
\hline Fercayl injection $\left(\mathrm{mg} \mathrm{mL}^{-1}\right)$ & $45.12 \pm 0.21$ & $45.20 \pm 0.07$ & 10.05 & 0.67 & 0.542 \\
\hline Hydroferrin syrup $\left(\mathrm{mg} \mathrm{mL}^{-1}\right)$ & $55.72 \pm 0.98$ & $56.56 \pm 0.23$ & 17.68 & 1.45 & 0.220 \\
\hline Theragran multivitamin (mg per tablet) & $64.95 \pm 0.62$ & $65.45 \pm 0.32$ & 3.88 & 2.11 & 0.102 \\
\hline
\end{tabular}

${ }^{a}$ Collected downstream, approximately $1 \mathrm{~km}$ from the Qasr El Nile Bridge on March 10, 2017; $(\mathrm{pH}=8.10) .{ }^{b}$ Collected from the exit pipe of 101 Chem Lab. 
analysis of natural and waste waters and three pharmaceutical samples: Fercayl injection solution, Hydroferrin syrup, and Theragran multivitamin tablets. Table 1 shows the results of iron determination obtained using the proposed DIBA and standard phenanthroline-based spectrophotometric methods. At the $95 \%$ confidence level, the Student's $t$-test and the $F$-test could not detect any significant difference between the means and variances of the two methods. ${ }^{38}$

\section{Conclusion}

In conclusion, we report a homemade, low-cost, and portable camera-based platform with Yxy signalling and its application to the assessment of iron. Our inexpensive and simple design is free from special light sources, lenses, slits, mirrors, filters, gratings, special sample compartments, photodiodes, photomultipliers, and signal amplifiers. In principle, it requires only a cuvette, a camera (or mobile phone or scanner), and a white paper as a diffuser. Moreover, it can measure absorption, reflectance, and/or luminescence without any additional instrumentation or software, indicating its potential as an excellent competitor to sophisticated laboratory spectrophotometers and spectrofluorometers. This would be very promising, especially in resource-limited environments. In addition, it can in principle analyse not only large volume liquid samples but also nano- or microlitre droplet and thin-film samples of transparent, non-transparent, solid, turbid or opaque dispersion samples where common spectrophotometers and spectrofluorometers usually require special attachment units. With our platform, a comparison of various non-uniform (RGB, greyscale, inverted greyscale, CMY, tristimulus XYZ, and Yxy) signals revealed that the $A_{x}$ and/or $A_{y}$ colour absorbance parameters of the Yxy colour space yielded calibration graphs with excellent linearity, slope-to-intercept ratio, and LOD irrespective of the colour and nature of the reaction system. Moreover, the linearity and sensitivity of the calibration lines were independent of the type and resolution of the imagecapturing device. The excellent performance of the platform was demonstrated by its application to the assessment of iron in natural and waste waters and pharmaceutical samples. Statistical treatment of the analytical results revealed no significant difference between the data obtained using our platform and the sophisticated Shimadzu UV-1601 PC spectrophotometer.

\section{Conflicts of interest}

There are no conflicts to declare.

\section{Notes and references}

1 N. A. Rakow, A. Sen, M. C. Janzen, J. B. Ponder and K. S. Suslick, Angew. Chem., Int. Ed. Engl., 2005, 44, 45284532.

2 R. C. Evans and P. Douglas, Anal. Chem., 2006, 78, 56455652.
3 A. W. Martinez, S. T. Phillips, M. J. Butte and G. M. Whitesides, Angew. Chem., Int. Ed. Engl., 2007, 46, 1318-1320.

4 A. Scheeline, Appl. Spectrosc., 2010, 256A-268A, http:// scheeline.scs.illinois.edu/ asweb/CPS/, accessed 08-Jan2018.

5 F. R. d. Souza, G. F. Duarte Junior, P. d. T. Garcia and W. K. T. Coltro, Quim. Nova, 2014, 37, 1171-1176.

6 H. Kudo, K. Yamada, D. Watanabe, K. Suzuki and D. Citterio, Micromachines, 2017, 8, 127-138.

7 P. Masawat, A. Harfield, N. Srihirun and A. Namwong, Anal. Lett., 2016, 50, 173-185.

8 S. K. Vashist, T. van Oordt, E. M. Schneider, R. Zengerle, F. von Stetten and J. H. Luong, Biosens. Bioelectron., 2015, 67, 248-255.

9 E. H. Doeven, G. J. Barbante, E. Kerr, C. F. Hogan, J. A. Endler and P. S. Francis, Anal. Chem., 2014, 86, 2727-2732.

10 S. J. E. Andrade, M. B. Lima, I. S. Barreto, W. S. Lyra, L. F. Almeida, M. C. U. Araujo and E. C. Silva, Microchem. J., 2013, 109, 106-111.

11 J. A. V. A. Barros, F. M. d. Oliveira, G. d. O. Santos, C. Wisniewski and P. O. Luccas, Anal. Lett., 2016, 50, 414430.

12 L. J. Wang, R. Sun, T. Vasile, Y. C. Chang and L. Li, Anal. Chem., 2016, 88, 8302-8308.

13 B. M. Jayawardane, I. D. McKelvie and S. D. Kolev, Anal. Chem., 2015, 87, 4621-4626.

14 D. C. Christodouleas, A. Nemiroski, A. A. Kumar and G. M. Whitesides, Anal. Chem., 2015, 87, 9170-9178.

15 Q. Wei, R. Nagi, K. Sadeghi, S. Feng, E. Yan, S. J. Ki, R. Caire, D. Tseng and A. Ozcan, ACS Nano, 2014, 8, 1121-1129.

16 S. A. Klasner, A. K. Price, K. W. Hoeman, R. S. Wilson, K. J. Bell and C. T. Culbertson, Anal. Bioanal. Chem., 2010, 397, 1821-1829.

17 B. Pang, C. Zhao, L. Li, X. Song, K. Xu, J. Wang, Y. Liu, K. Fu, H. Bao, D. Song, X. Meng, X. Qu, Z. Zhang and J. Li, Anal. Biochem., 2018, 542, 58-62.

18 W.-H. Liang, C.-H. Chu and R.-J. Yang, Talanta, 2015, 145, 611.

19 A. W. Martinez, S. T. Phillips, E. Carrilho, S. W. Thomas III, H. Sindi and G. M. Whitesides, Anal. Chem., 2008, 80, 36993707.

20 J. C. Ye, N. Li, Y. Lu, J. Cheng and Y. C. Xu, Anal. Methods, 2017, 9, 2464-2471.

21 V. M. Ivanov, V. N. Figurovskaya and Y. I. Shcherbakova, Moscow Univ. Chem. Bull., 2013, 68, 191-195.

22 V. M. Ivanov, O. V. Monogarova and K. V. Oskolok, J. Anal. Chem., 2015, 70, 1165-1178.

23 B. Wang, Z. Lin and M. Wang, J. Chem. Educ., 2015, 92, 733736.

24 H. Asano and Y. Shiraishi, Anal. Chim. Acta, 2015, 883, 55-60. 25 P. Rattanarat, W. Dungchai, D. Cate, J. Volckens, O. Chailapakul and C. S. Henry, Anal. Chem., 2014, 86, 3555-3562.

26 P. Rattanarat, W. Dungchai, D. M. Cate, W. Siangproh, J. Volckens, O. Chailapakul and C. S. Henry, Anal. Chim. Acta, 2013, 800, 50-55. 
27 L. F. Capitan-Vallvey, N. Lopez-Ruiz, A. Martinez-Olmos, M. M. Erenas and A. J. Palma, Anal. Chim. Acta, 2015, 899, 23-56.

28 G. G. Morbioli, T. Mazzu-Nascimento, A. M. Stockton and E. Carrilho, Anal. Chim. Acta, 2017, 970, 1-22.

29 V. V. Apyari, M. V. Gorbunova, A. I. Isachenko, S. G. Dmitrienko and Y. A. Zolotov, J. Anal. Chem., 2017, 72, 1127-1137.

30 R. W. G. Hunt and M. R. Pointer, Measuring colour, John Wiley \& Sons, Ltd, United Kingdom, Fourth edn, 2011.

31 http://colormine.org/color-converter, accessed 8-Jan-2018.

32 Z. Marczenko and M. Balcerzak, Separation, Preconcentration and Spectrophotometry in Inorganic Analysis, Amsterdam, The Netherlands, 2000.

33 Method 3500-Fe B, in Standard Methods for The Examination of Water and WasteWater, ed. E. W. Rice, R. B. Baird, A. D.
Eaton and L. S. Clesceri, American Public Health Association, Washington, DC, 22nd edn, 2011.

34 Method 315 B, in EPA's Sampling and Analysis Methods, ed. L. H. Keith, CRC Press, Inc., United States of America, 2nd edn, 1996.

35 Methods 937.03, 970.13, in Official Methods of Analysis, AOAC INTERNATIONAL, Gaithersburg, MD, 17th edn, 2000.

36 P. F. Collins, H. Diehl and G. F. Smith, Anal. Chem., 1959, 31, 1862-1867.

37 J. T. Mitchell-Koch, K. R. Reid and M. E. Meyerhoff, J. Chem. Educ., 2008, 85, 1658-1659.

38 J. N. Miller and J. C. Miller, Statistics and Chemometrics for Analytical Chemistry, Pearson Education Limited, Harlow, England, Sixth edn, 2010. 